health care plans (EHCPs). They provide concise clinical recommendations for use in an emergency while also aiming to empower patients. There is however no research evaluating the views of palliative care patients on EHCPs.

Objectives The objective of this pilot study was to describe the views of community-based palliative care patients in USA on EHCPs.

Methods The study was conducted in February 2018 with community based palliative care patients in Greater Cincinnati, Ohio, USA. Patients were recruited if they had completed an EHCP and it had been valid for at least 1 month. There was 2 parts to the study: a written quantitative survey and a face to face interview. The study was approved by the health system's Institutional Review Board.

Results Demographics - 7 out of 10 patients eligible participated in the study (Survey 6 patients, Interview 3 patients).

Survey Results $100 \%$ patients felt an EHCP guided them during an emergency and they would recommend them to other patients. $100 \%$ patients did not share their EHCP with other professionals.

Interview Themes: Patients were overwhelmingly positive of EHCPs: they helped in preventing unwanted hospitalisation and aided symptom management. They described the time to write an EHCP as reasonable.

Conclusion The results of this study demonstrate that patients use EHCPs to guide their care during an emergency. This reaffirms the place of EHCPs in the advance care planning process. Patients did not share EHCPs with other professionals but this may be due to EHCPs being new to the health system. Finally, as this was a pilot study, further large scale research is required to further determine patients' views.

\section{A SIMPLE ACT OF KINDNESS CAN DO MORE THAN MEDICINE: PALLIATIVE CARE TRAINING FOR COMMUNITY HEALTH WORKERS IN RURAL MYANMAR}

Kerry E Waterfield. Health Education England North East

\subsection{6/spcare-2020-PCC.121}

Introduction Chin State, a very remote and mountainous state, is the most impoverished in Myanmar. Health and Hope has trained 834 community health care workers (CHWs) from over 550 villages across Chin and neighbouring Rakhine state to address basic healthcare needs in their communities. The current project involves the selection and training of Area Coordinators (ACs) to provide support, ongoing training and supplies to a group of CHWs in their geographical area. Palliative Care is underdeveloped and resourced in Myanmar, which ranked 76th out of 80 countries in the Economist 'Quality of death' index. There is no known provision in Chin State.

Method A half day workshop was delivered to 23 ACs which aimed

- To improve understanding of issues facing patients at the end of life

- To introduce palliative care and a holistic approach

- To improve confidence in looking after patients at the end of life

Results None of the participants had previously heard of palliative care. ACs reported that most had looked after patients at the end of their lives with many difficult and distressing stories involving huge suffering. Verbal feedback at the end of the workshop demonstrated that they had acquired a good understanding of palliative care and a holistic approach in several key areas and had improved in their confidence of caring for patients at the end of their lives.

Conclusion There is a significant need for palliative care in Myanmar, due to a high level of serious health related suffering and poor current provision. A short training package delivered to CHWs improved understanding of palliative care and confidence in looking after dying patients. Additional education, training and resources could further equip the team with the potential to make a significant impact on the lives and deaths of those in Chin and neighbouring states in Myanmar.

\section{Pain | posters $102-105$}

\section{USE OF TAPENTADOL FOR PAIN MANAGEMENT IN PALLIATIVE CARE: A RETROSPECTIVE CASE NOTE ANALYSIS OF HOSPITAL INPATIENTS}

Poppy Brown, Jessica Lee, Amara C Nwosu, Andrew Dickman, Sarika Hanchanale. School of Medicine - University of Liverpool, Liverpool University Hospitals NHS Foundation Trust, Palliative Care Institute Liverpool, Marie Curie Hospital Liverpool

\subsection{6/spcare-2020-PCC.122}

Background Tapentadol is a relatively new centrally-acting analgesic medication. Tapentadol is approved for the treatment of severe chronic pain and may reduce the need for strong opioids and reduce side effect burden. However, there is limited data about the use of tapentadol in palliative care. This project aimed to evaluate use of tapentadol in a hospital specialist palliative care unit.

Methods This project included the following: 1) Retrospective case note review of tapentadol use in all inpatients receiving care in a UK hospital specialist palliative care unit over a 12 month period. The primary outcome was whether pain improved following tapentadol use. The following information was collected: reason for initiation, initial dose, titration regime, side-effects, treatment duration, discontinuation reason and quality of discharge information.

2) Anonymised survey of healthcare professionals working within the palliative care team regarding their knowledge about, and use of, tapentadol.

Results Tapentadol was used in 10 individuals. Mean age 65 years; $6(60 \%)$ were male and $4(40 \%)$ were female, all patients had cancer. There was an improvement of pain in 5 $(50 \%)$ patients; this was identified through electronic clinical records. Nine (90\%) received a starting dose of $50 \mathrm{mg}$ BD. The final dose given was $50 \mathrm{mg} \mathrm{BD}$ in $5(50 \%)$ people, followed by $100 \mathrm{mg} \mathrm{BD}$ in $4(40 \%)$ and $150 \mathrm{mg} \mathrm{BD}$ in one $(10 \%)$. Tapentadol was discontinued in five $(50 \%)$ due to side effects, inability to swallow and renal failure. Eleven healthcare professionals completed the questionnaire. Of the respondents, $5(45 \%)$ had prescribed or given advice on prescribing tapentadol and $10(91 \%)$ indicated that they would like further training.

Conclusion Tapentadol was used in small number of individuals who all had cancer pain. Our data suggests that tapentadol may be beneficial in palliative care. More research and staff education is important to ensure effective use. 